\title{
BMJ Open Impact of Bariatric surgery on EmbrYONic, fetal and placental Development (BEYOND): protocol for a prospective cohort study embedded in the Rotterdam periconceptional cohort
}

Katinka M Snoek (D) , ${ }^{1}$ Régine P M Steegers-Theunissen (D) , ${ }^{1}$ René A Klaassen, ${ }^{2}$ Joop S E Laven, ${ }^{3}$ Sam Schoenmakers ${ }^{1}$

To cite: Snoek KM, Steegers-Theunissen RPM, Klaassen RA, et al. Impact of Bariatric surgery on EmbrYONic, fetal and placental Development (BEYOND): protocol for a prospective cohort study embedded in the Rotterdam periconceptional cohort. BMJ Open 2021;11:e051110. doi:10.1136/ bmjopen-2021-051110

- Prepublication history for this paper is available online. To view these files, please visit the journal online (http://dx.doi. org/10.1136/bmjopen-2021051110).

Received 11 March 2021 Accepted 19 August 2021

\section{Check for updates}

(C) Author(s) (or their employer(s)) 2021. Re-use permitted under CC BY-NC. No commercial re-use. See rights and permissions. Published by BMJ.

${ }^{1}$ Department of Obstetrics and Gynaecology, Erasmus Medical Centre, Rotterdam, The Netherlands

${ }^{2}$ Department of Surgery, Maasstad Hospital, Rotterdam, The Netherlands

${ }^{3}$ Division of Reproductive Endocrinology and Infertility, Department of Obstetrics and Gynaecology, Erasmus Medical Centre, Rotterdam, The Netherlands

Correspondence to Dr Sam Schoenmakers; s.schoenmakers@erasmusmc.n

\section{ABSTRACT}

Introduction The worldwide obesity epidemic has resulted in a rise of bariatric surgery in women of reproductive age, which can lead to 'iatrogenic undernutrition'. Long-lasting undernutrition can affect maternal health, pregnancy outcomes and offspring. We hypothesise that embryonic and placental growth are impaired in pregnancies after bariatric surgery due to the changed nutritional and microbiome dynamics. Therefore, our aim is to conduct the Bariatrics and EmbrYONic Development (BEYOND) study to investigate parameters of maternal nutritional and health status after bariatric surgery, both periconceptionally and during pregnancy, particularly concentrating on embryonic and fetal growth trajectories as well as placental development.

Methods and analysis We designed a single-centre prospective, observational cohort, which investigates the iatrogenic nutritional and health status of women after bariatric surgery, periconceptionally and during pregnancy. The BEYOND study is embedded in the Rotterdam Periconceptional Cohort, a tertiary hospitalbased birth cohort study. Eligible participants are women planning pregnancy or $<12+0$ weeks pregnant, $\geq 18$ and $\leq 45$ years of age, who have undergone bariatric surgery (cases) or without prior bariatric surgery (controls) and their male partners. Medical charts will be reviewed and questionnaires regarding general health, lifestyle and food intake will be collected. Moreover, we will perform serial three-dimensional ultrasounds to assess embryonic growth and placental development and two-dimensional ultrasounds for fetal growth assessment. The microbiome, including the virome, and blood samples will be sampled during the preconception period and in each trimester. Multivariable linear mixed model analyses will be used to assess the associations between bariatric surgery and pregnancy outcomes.

Ethics and dissemination This proposal was approved by the Medical Ethics Committee from the Erasmus MC, Rotterdam, The Netherlands. Study results will be submitted for publication in high-impact journals, presented at scientific conferences, implemented into guidelines and communicated through the Erasmus MC and collaborating partners.

\section{Strengths and limitations of this study}

- The prospective and longitudinal assessment of postbariatric women preconceptionally, during and after pregnancy provides an in-depth knowledge of the reproductive trajectory of these women.

- The prospective design of this study allows us to analyse vitamin levels after vitamin supplement use in postbariatric women, which gives the opportunity to define individualised and tailored recommendations for additional vitamin supplementation.

- The three-dimensional ultrasound examinations during the first trimester provide detailed information regarding embryonic, fetal and placental growth and development in postbariatric women for the first time.

Due to ethical reasons, the design of this study is not a randomised controlled trial, being the gold standard for clinical research.

Trial registration number NL8217 (www.trialregister.nl).

\section{INTRODUCTION}

The incidence of obesity, which is defined as a body mass index (BMI) of $\geq 30 \mathrm{~kg} / \mathrm{m}^{2}$, is increasing worldwide, including in women of reproductive age. ${ }^{1}$ Importantly, prepregnancy and periconceptional obesity are now well-established independent risk factors for fetomaternal complications and disease risks in offspring as well as for women during the life course (e.g. hypertension and type II diabetes mellitus) ${ }^{2-5}$ The risks along the life course can be significantly reduced by achieving prepregnancy weight loss and as such a reduction in BMI. Weight loss in obesity can be accomplished by lifestyle and nutritional changes, pharmaceutical therapies or medical weight loss interventions 
programmes, bariatric surgery or a combination of these interventions.

\section{Bariatric surgery and related weight loss}

Bariatric surgery is an effective surgical solution to quickly lose excess weight and reach a healthier longterm weight. ${ }^{6-10}$ Patients qualify for bariatric surgery if they have a BMI $>40 \mathrm{~kg} / \mathrm{m}^{2}$ or a BMI above $35 \mathrm{~kg} / \mathrm{m}^{2}$ along with at least one obesity-related comorbidity such as diabetes mellitus. ${ }^{11-15}$ There are different types of bariatric surgery, based on their endocrine, metabolic and (patho)physiological consequences. Malabsorptive procedures lead to impaired uptake of nutrients, whereas restrictive procedures mainly decrease food intake. However, a sleeve gastrectomy, which is often considered a restrictive procedure, also has endocrine and metabolic effects. ${ }^{16}$ Since bariatric surgery leads to fast, excessive and-most importantly-long-term weight loss, preconception bariatric surgery in women of reproductive age can diminish the prevalence of obesity-related adverse maternal and fetal outcomes. ${ }^{17-21}$ Unfortunately, due to fast and excessive weight loss resulting from gastrointestinal anatomical changes of preconception bariatric surgery, iatrogenic malnutrition can also increase the incidence of adverse pregnancy and perinatal outcomes, such as intrauterine growth restriction and congenital vitamin deficiencies in neonates. Growth restriction seems to be mainly present after malabsorptive surgery, as nutritional deficiencies occur more often after this type of bariatric procedure. ${ }^{18} 19$

\section{Bariatric surgery and nutritional status}

Gastrointestinal surgical changes after bariatric surgery can cause malabsorption and iatrogenic malnutrition, including vitamin deficiencies. A high incidence of vitamin deficiencies has been reported in patients after bariatric surgery. ${ }^{22}$ Most reported deficiencies during the first trimester after bariatric surgery are, among others, vitamin B1, folate and vitamin D. ${ }^{23-26}$ However, ample research has been performed to map, treat and investigate consequences of these vitamin deficiencies in these women during the periconception period, with potential consequences for embryonic growth and development. $^{27}{ }^{28}$ Vitamin B1 (thiamin) is needed for the synthesis of myelin and involved in mitochondrial and synaptosomal membranes and is vital for fetal neural and brain development. ${ }^{29}$ Vitamin B1 deficiency impacts intrauterine growth, causing growth restriction, while vitamin $\mathrm{D}$ deficiency can result in postnatal motor development disorders. ${ }^{30}$ Folate deficiency can lead to impaired oocyte quality, subfertility, congenital malformations and several placenta-related pregnancy complications. ${ }^{31-34}$ Postsurgical multivitamin supplementation after bariatric surgery is highly dosed to correct for the anticipated deficiencies and has only been developed for the non-pregnant patient. Hereby, the used dosage regimen can lead to supraphysiological levels, with potential teratogenic levels and detrimental effects for the developing fetus. ${ }^{35}$

\section{Nutritional status and mechanisms}

An adequate maternal nutritional and vitamin status is essential for optimal fetal development. ${ }^{27-34}$ Barker et al were the first to suggest that maternal nutrition during pregnancy directs and programmes fetal development in utero, the so-called 'Developmental Origins of Health and Disease' dogma. ${ }^{36}$ For example, maternal undernutrition has been associated with the susceptibility of developing non-communicable diseases in later life by fetal programming, whereas adequate nutritional health has been shown to reduce the odds of obesity in the offspring. ${ }^{37-39}$

\section{One-carbon metabolism and the microbiome}

Obesity is also linked with an imbalanced one-carbon metabolism. ${ }^{41}$ The one-carbon metabolism is essential for DNA methylation and gene expression and plays a vital role in physiological processes such as biosynthesis, cell division and proliferation. ${ }^{42}$ Especially, embryonic and fetal growth are characterised by a need for rapid cellular multiplication, division and proliferation and, therefore, the one-carbon metabolism is crucial for embryonic and fetal development. Key players of the onecarbon metabolism such as folate are either provided by diet, including vitamin supplements or as by-products of the bacterial metabolism. The gut microbiota, collectively known as the gut microbiome, includes bacteria (bacteriome) as well as viruses (virome). Importantly, periconception maternal nutritional status and alterations, such as bariatric surgery, can influence general cellular function, metabolism and the gut microbiome. Bariatric surgery can hereby affect fetal growth and metabolism. Bariatric surgery is besides anatomical gastrointestinal tract changes also associated with lifestyle and dramatic gut microbiome changes. ${ }^{44}$ Therefore, current information is needed regarding the influence of bariatric surgery on maternal nutritional status, the dynamics and composition of the microbiome and one-carbon metabolism and their combined effect on embryonic, fetal and placental development.

\section{METHODS AND ANALYSIS}

We designed a prospective single-centre observational cohort study, which is embedded in the Rotterdam Periconceptional cohort (Predict study, MEC 2004-227), to investigate postbariatric women both before and during pregnancy with the aim to study the association between bariatric surgery and embryonic, fetal and maternal health.

\section{Study design}

Setting

All study subjects will be included at the outpatient clinic of the Erasmus MC, University Medical Centre. Eligible cases will be provided with a study leaflet by healthcare professionals at the Bariatric Centre, Obstetric or Fertility outpatient clinic, after which they will be asked to participate. Women and their partners will be referred from the 
moment they are planning pregnancy and/or before $12+0$ weeks gestation of pregnancy. Controls are selected from the Predict study. ${ }^{45}$ With the BEYOND study, serial embryonic and fetal growth parameters, the microbiome and blood samples will be documented prospectively. The primary objective of this study is to investigate the association between preconception maternal bariatric surgery and subsequent embryonic, fetal and placental growth. We hypothesise that bariatric surgery impairs embryonic and fetal growth due to postsurgical nutritional deficiencies. Secondary objectives include the investigation of associations between preconception bariatric surgery and clinical maternal and pregnancy outcomes, one-carbon metabolism effects such as homocysteine levels, the microbial composition, placental growth and development and maternal lifestyle.

\section{Study population}

In order to be eligible to participate in this study, women have to be $\geq 18$ and $\leq 45$ years of age, either planning pregnancy or $\leq 12+0$ weeks pregnant of a singleton pregnancy. Their male partners will also be included. They must have sufficient understanding of the Dutch language and must be willing to give written informed consent. Cases must have had bariatric surgery, excluding a gastric banding procedure that has been deflated or removed prior to pregnancy. Controls will be women who have not undergone bariatric surgery and will be selected from the current Predict study.

\section{Study procedures}

The duration of the study will be 48 months and will include different measurements and procedures (figure 1).

\section{Ultrasound scans}

At 7, 9 and 11 weeks gestational age (GA), the gestational sac, embryo and placenta are depicted in 3D ultrasound scans using a GE Voluson E8 or E10 Expert system with a 6-12 megaherz transvaginal probe and 4D View software (General Electric Medical Systems, Zipf, Austria). We will perform 3D ultrasound scans that focus on embryonic and placental development including crown-rump length (CRL), embryonic volume, brain structures and the gestational sac, placenta and yolk sac. Moreover, we will evaluate the placental vasculature, pulsatility index and resistance index of the uterine arteries at 9 and 11 weeks GA using ultrasound Doppler.

At 22-24 weeks GA and 30-32 weeks GA, fetal growth will be assessed by the growth parameters head circumference, biparietal diameter, abdominal circumference and femur length. Moreover, ultrasound Dopplers of the umbilical arteries, middle cerebral artery and uterine arteries will be performed.

All scans will be made with standard settings of the ultrasound machine: pulse repetition frequency of 0.6 $\mathrm{kz}$, gain -2.0 , quality 'high', wall motion filter 'low'. The obtained 3D data sets will be stored as Cartesian (rectangular) volumes. Moreover, we will follow the as low as reasonably achievable principle to ensure safe ultrasound examinations.

Pulsed wave Doppler is a standardised additional modality of ultrasound imaging to quantify blood flow. Placental and uterine blood flow will be quantified as expressed by the resistance index as well as by the pulsatility index. Hence, the use of pulsed wave Doppler signal during ultrasound enables us to perform a non-invasive
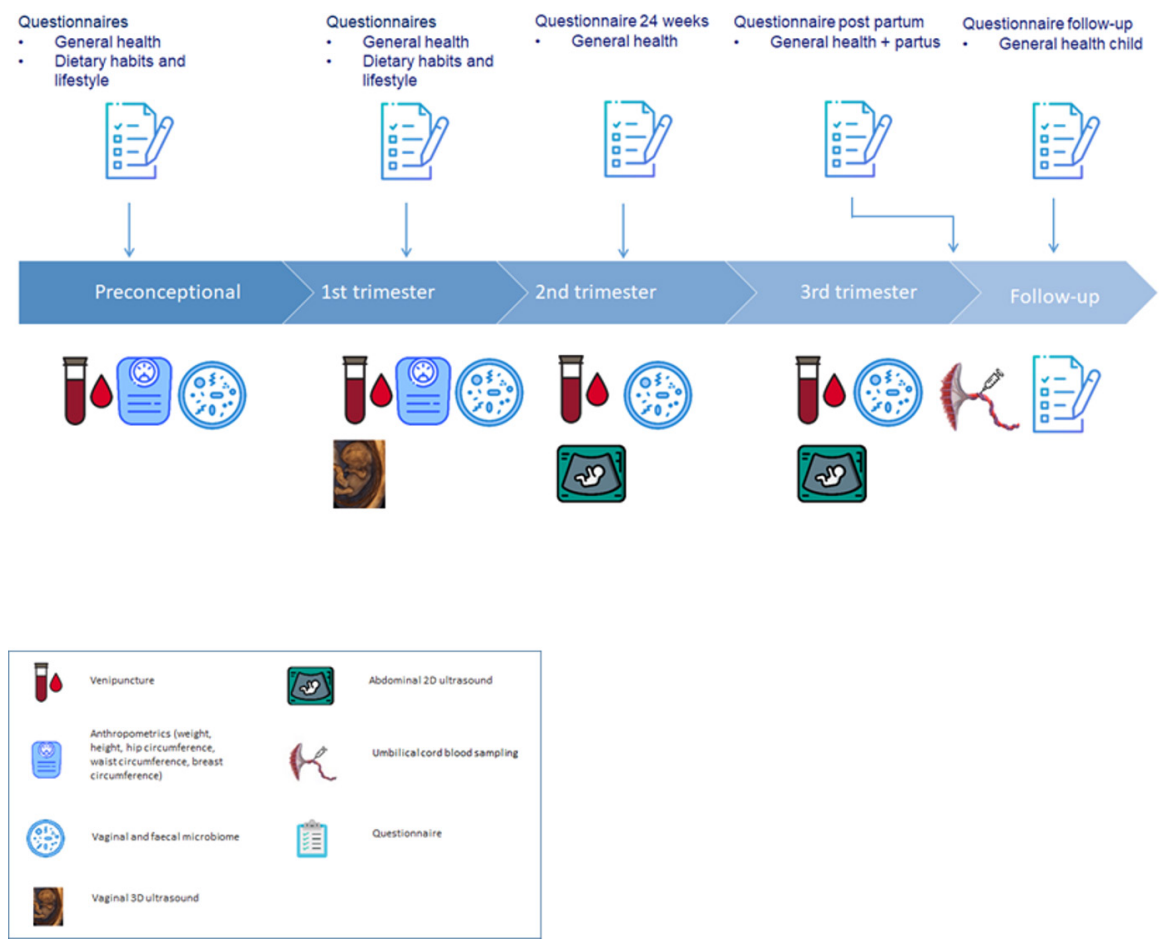

Figure 1 Enrolment, collection of data and materials and follow-up. 
measurement of the blood flow and subsequently to detect changes in flow.

\section{Questionnaires}

Women and their male partners will fill out a selfadministered, validated food frequency questionnaire regarding food intake of the previous 4 weeks. ${ }^{46-48}$ Portion sizes are quantified by this questionnaire.

Moreover, preconceptionally and during the first trimester, a general questionnaire will be filled out including geographical background, education and lifestyle. At 24 weeks GA, a questionnaire is filled out regarding information about folic acid intake, any vitamin supplementation, lifestyle behaviour, prenatal screening, results of the fetal anomaly scan (around 20 weeks of gestation) and previous pregnancy outcome. Postpartum, women are asked to fill out a questionnaire regarding neonatal health. As follow-up 1-year postpartum, women are asked to fill out the last questionnaire regarding congenital malformations, general health and medical history of the offspring.

\section{Blood samples}

Preconceptionally and during all three trimesters of pregnancy, two blood samples of $10 \mathrm{~mL}$ in a vacutainer EDTA tube and one blood sample of $8.5 \mathrm{~mL}$ in a serum tube will be drawn. These samples will be centrifuged directly and separated into serum, plasma, whole blood and buffy coat aliquots. Parameters of the one-carbon metabolism such as homocysteine serum levels will be measured.

Umbilical cord blood is collected at delivery in one EDTA tube $(10 \mathrm{~mL})$ and a separator tube $(10 \mathrm{~mL})$. After this process, all blood samples will be stored at $-80^{\circ} \mathrm{C}$ at the Erasmus MC for this study and future research.

\section{Microbiome samples}

Preconceptionally and during all three trimesters of pregnancy, the vaginal and faecal microbiome, including the virome, will be sampled. Vaginal and faecal samples will be self-collected by the patient. After collection, the vaginal and faecal samples will be stored at $-80^{\circ} \mathrm{C}$ until processing. The microbiome samples will also be collected prospectively in the Predict study cohort.

\section{Sample size}

We performed a simulation study to calculate the sample size and compared the profile of embryonic volume in the simulation study with controls who had not undergone bariatric surgery using a multivariate test. The relationship between bariatric surgery and the outcome could be influenced by several confounders. In the sample size calculation, we focused on BMI and age of the mother, which are the most important confounders. In the control group, the mean of these variables is respectively $25.5 \mathrm{~kg} / \mathrm{m}^{2}\left(\mathrm{SD}=4.9 \mathrm{~kg} / \mathrm{m}^{2}\right)$ and 31.9 years $(\mathrm{SD}=4.5$ years). In the bariatric surgery group, we postulate that the mean BMI is $30.1 \mathrm{~kg} / \mathrm{m}^{2}$ at conception and the mean age is 31 years with identical SD as in the non-bariatric surgery group, based on recent research. ${ }^{49-59}$ We aimed to detect a difference in the cube root embryonic volumes of 0.059 millimetres as this is the difference between adequate and inadequate folate state. ${ }^{60}$ The SD of the cube root embryonic volume given the covariates is 0.105 . Simulations showed that we need at least 50 patients to detect a difference with $80 \%$ power and an alpha of 0.05 . Given an average miscarriage rate of $10 \%$ and an estimate of a $30 \%$ dropout, we will need to include 80 pregnant patients. With an average chance to conceive within a year of $84 \%$, this results in a total number of 95 preconceptional patients. ${ }^{61}$

\section{Statistical analysis}

To assess the association between preconception bariatric surgery and embryonic growth trajectories measured by embryonic volume, CRL and placental volume, we will perform multivariable linear mixed model analyses using patients without bariatric surgery as a control group. By using a mixed model, we consider a correlation between the observations within the same pregnancy. Cube root transformation will be used to investigate if this results in linearity with GA and is, therefore, a constant variant independent of GA. We will first perform a univariate analysis, in which we adjust for GA only. After this, we will enter all covariates that are significantly correlated with bariatric surgery. The fully adjusted model will be made after stepwise elimination of all covariates with $p$ values above the 20th percentile. Potential confounders will be identified by performing a literature search, by using analysis of variance and by calculating Spearman correlation coefficients for the other maternal characteristics such as, but not limited to, maternal BMI, parity, smoking, age, mode of conception and fetal sex.

Missing data will be handled by multiple imputation. A $\mathrm{p}$ value $<0.05$ will be considered as statistically significant.

The primary outcome parameter is defined as the embryonic growth trajectory assessed by serial embryonic volumes. Since all other outcome parameters are secondary or descriptive, no correction for multiplicity will be performed. For categorical variables such as smoking and alcohol use, we will use descriptive characteristics.

To test the influence of vitamin deficiencies and excesses related to supplement usage, distribution will be tested using student's t-test for normal distributions, Kruskal-Wallis test for non-parametric distributions and $\chi^{2}$ or exact tests for categorical data.

To assess the relationship between maternal parameters such as pregnancy outcome, bariatric surgery and longitudinal changes in vaginal and faecal microbiome and blood samples, we will test whether the data are normally distributed by using the Shapiro-Wilks normality test. The independent samples t-test and Mann-Whitney U test will be used for continuous data.

Continuous, normally distributed variables will be presented as mean with SD and variables with a skewed distribution as median with the range. Categorical variables will be presented as count and proportions. Differences between women who have undergone bariatric 
surgery and women who have not undergone bariatric surgery will be compared, correcting for differences in BMI at conception.

To assess the association between preconception bariatric surgery and embryonic growth trajectories by CRL, we will perform multivariable linear mixed model analyses. By using linear mixed model analyses, we consider a correlation between the observations within the same pregnancy.

We will repeat analyses in the subgroups of in vitro fertilisation/intracytoplasmic sperm injection pregnancies only and in subgroups of different types of bariatric surgery if the groups are large enough. Birth weight will be compared between groups by taking GA at birth into account using linear regression analysis.

\section{DATA STATEMENT}

The current study will abide by the principles of the Declaration of Helsinki (October 2013) and all national and European Union guidelines. The study protocol has been approved by the local Medical Ethics Committee of the Erasmus Medical Centre (MEC 2019-0518) and has been registered in the Dutch Trial Register (www.trialregister.nl). All participants will only be included after informed consent.

Data will be pseudoanonymised in order to guarantee the privacy of the patients by assigning a study-ID. We will keep a strictly confidential mapping from their local patient-ID to the study-ID. Patient data will be stored on a separate protected research storage platform. Access will be limited to authorised medical personnel. All research data will be retained and stored in the study database at the Erasmus MC.

\section{PATIENT AND PUBLIC INVOLVEMENT}

Patients were not involved in the development of the research question, outcome measures or study design, but the project will be communicated to patients by using the internal and external communication means of the Erasmus MC, University Medical Centre, collaborating partners, such as institutional and patient association websites, social media, magazines and the yearly news letter from the Rotterdam periconceptional cohort.

\section{ETHICS AND DISSEMINATION}

This study was registered prospectively in December 2019 after ethical approval by the Medical Ethics Committee from the Erasmus MC, Rotterdam, The Netherlands. We will disseminate our study results by publishing papers in high-impact journals, presentations at scientific conferences and implement the results into local and (inter) national guidelines.

\section{RESEARCH IMPLICATIONS}

The results of this study will provide detailed information about embryonic, fetal and placental growth and development during pregnancy in postbariatric women. Furthermore, this study will elucidate maternal periconception health and pregnancy course, embryonic and fetal growth trajectories and placental development in women after bariatric surgery. We also aim to gain more insight into the underlying pathways, leading to iatrogenic malnutrition after bariatric surgery and the resulting weight loss, how these circumstances influence the local microbiome and how we could potentially prevent them by timely correction. Particularly, it is of utmost importance to investigate the effects on embryonic, fetal and placental development. With the results of the BEYOND study, severely obese women planning bariatric surgery and pregnancy can be counselled more precisely concerning the (dis)advantages of bariatric surgery related to the periconception period, pregnancy and placental, embryonic, fetal and neonatal health. Therefore, improved periconception healthcare counselling can be provided, allowing for better informed decision-making about bariatric surgery and a future pregnancy.

In general, we can equip healthcare providers with patient-tailored advice for obese women of reproductive age during the periconception period. By analysing vitamin ranges from the preconception period and all pregnancy trimesters, we will be able to map both the incidence of vitamin deficiencies and excesses due to (over)correction of deficiencies and supraphysiological vitamin supplementation. Hence, we intend to gain insight into possible teratogenicity of high-dose vitamin supplementation. Besides, quantitative and qualitative information about dietary intake will be retrieved from the food frequency questionnaires and will provide insight into possible dietary issues in these patients. This can contribute to healthcare improvement delivered by dietitians and other healthcare professionals for postbariatric pregnant patients. The results of our analyses will give insight into the incidence of vitamin deficiencies and excesses after vitamin supplementation in postbariatric women and will allow us to provide personalised counselling regarding optimal vitamin supplementation for these women. Therefore, periconception health can be improved, resulting in improved fetal, placental, neonatal and maternal health along the life course.

Contributors KMS, SS and RS-T designed the study. KMS will be executing the research with supervision from SS, RS-T and JSEL. RAK is involved in the retrospective part of this study. RS-T is the principal investigator in this study and SS is the principal coordinator. All authors have read and approved the final manuscript.

Funding The authors have not declared a specific grant for this research from any funding agency in the public, commercial or not-for-profit sectors.

Competing interests None declared.

Patient and public involvement Patients and/or the public were involved in the design, or conduct, or reporting, or dissemination plans of this research. Refer to the Methods section for further details.

Patient consent for publication Not required.

Provenance and peer review Not commissioned; externally peer reviewed. 
Open access This is an open access article distributed in accordance with the Creative Commons Attribution Non Commercial (CC BY-NC 4.0) license, which permits others to distribute, remix, adapt, build upon this work non-commercially, and license their derivative works on different terms, provided the original work is properly cited, appropriate credit is given, any changes made indicated, and the use is non-commercial. See: http://creativecommons.org/licenses/by-nc/4.0/.

\section{ORCID iDs}

Katinka M Snoek http://orcid.org/0000-0002-2732-9688

Régine P M Steegers-Theunissen http://orcid.org/0000-0002-4353-5756

\section{REFERENCES}

1 Finucane MM, Stevens GA, Cowan MJ, et al. National, regional, and global trends in body-mass index since 1980: systematic analysis of health examination surveys and epidemiological studies with 960 country-years and 9.1 million participants. The Lancet 2011;377:557-67.

2 Athukorala C, Rumbold AR, Willson KJ, et al. The risk of adverse pregnancy outcomes in women who are overweight or obese. BMC Pregnancy Childbirth 2010;10:56.

3 Avcı ME, Şanlıkan F, Çelik M, et al. Effects of maternal obesity on antenatal, perinatal and neonatal outcomes. J Matern Fetal Neonatal Med 2015;28:2080-3.

4 LifeCycle Project-Maternal Obesity and Childhood Outcomes Study Group, Voerman E, Santos S, et al. Association of gestational weight gain with adverse maternal and infant outcomes. JAMA 2019;321:1702-15.

5 Mission JF, Marshall NE, Caughey AB. Pregnancy risks associated with obesity. Obstet Gynecol Clin North Am 2015;42:335-53.

6 Colquitt JL, Pickett K, Loveman E, et al. Surgery for weight loss in adults. Cochrane Database Syst Rev 2014;90.

7 Chang S-H, Stoll CRT, Song J, et al. The effectiveness and risks of bariatric surgery: an updated systematic review and meta-analysis, 2003-2012. JAMA Surg 2014;149:275-87.

8 Maciejewski ML, Arterburn DE, Van Scoyoc L, et al. Bariatric surgery and long-term durability of weight loss. JAMA Surg 2016;151:1046-55

9 Wolfe BM, Kvach E, Eckel RH. Treatment of obesity: weight loss and bariatric surgery. Circ Res 2016;118:1844-55.

10 Dumon KR, Murayama KM. Bariatric surgery outcomes. Surg Clin North Am 2011;91:1313-38.

$11 \mathrm{NVv} \mathrm{H}$. Chirurgische behandeling van obesitas: federatie medisch specialisten, 2020. Available: https://richtlijnendatabase.nl/richtlijn/ chirurgische_behandeling_van_obesitas/startpagina_-_chirurgische_ behandeling van obesitas.html [Accessed 28 Oct 2020].

12 Fried M, Hainer V, Basdevant A, et al. Interdisciplinary European guidelines on surgery of severe obesity. Obes Facts 2008;1:52-9.

13 Mechanick JI, Youdim A, Jones DB. AACE/TOS/ASSMBS guidelines: clinical practice guideline for the perioperative nutritional, metabolic, and nonsurgical supp-port of the bariatric surgery patient-2013 update: cosponsored by american association of clinical endocrinologists, the obesity society, and american society for metabolic \& bariatric surgery. Endocrine Practice 2013;19.

14 Ridley N. Expert panel on weight loss surgery-executive report. Obes Res 2005;13:206-26.

15 Fried M, Yumuk V, Oppert JM, et al. Interdisciplinary European guidelines on metabolic and bariatric surgery. Obes Surg 2014;24:42-55.

16 Benaiges D, Más-Lorenzo A, Goday A, et al. Laparoscopic sleeve gastrectomy: more than a restrictive bariatric surgery procedure? World J Gastroenterol 2015;21:11804.

17 Maggard MA, Yermilov I, Li Z, et al. Pregnancy and fertility following bariatric surgery: a systematic review. JAMA 2008;300:2286-96.

18 Kwong W, Tomlinson G, Feig DS. Maternal and neonatal outcomes after bariatric surgery; a systematic review and meta-analysis: do the benefits outweigh the risks? Am J Obstet Gynecol 2018;218:573-80.

19 Akhter Z, Rankin J, Ceulemans D, et al. Pregnancy after bariatric surgery and adverse perinatal outcomes: a systematic review and meta-analysis. PLoS Med 2019;16:e1002866.

20 Shawe J, Ceulemans D, Akhter Z, et al. Pregnancy after bariatric surgery: consensus recommendations for periconception, antenatal and postnatal care. Obes Rev 2019;20:1507-22.

21 Al-Nimr RI, Hakeem R, Moreschi JM, et al. Effects of bariatric surgery on maternal and infant outcomes of Pregnancy-An evidence analysis center systematic review. J Acad Nutr Diet 2019;119:1921-43.

22 Bloomberg RD, Fleishman A, Nalle JE, et al. Nutritional deficiencies following bariatric surgery: what have we learned? Obes Surg 2005;15:145-54.
23 Davies DJ, Baxter JM, Baxter JN. Nutritional deficiencies after bariatric surgery. Obes Surg 2007;17:1150-8.

24 Bal BS, Finelli FC, Shope TR, et al. Nutritional deficiencies after bariatric surgery. Nat Rev Endocrinol 2012;8:544-56.

25 Xanthakos SA. Nutritional deficiencies in obesity and after bariatric surgery. Pediatr Clin North Am 2009;56:1105-21.

26 Falcone V, Stopp T, Feichtinger M, et al. Pregnancy after bariatric surgery: a narrative literature review and discussion of impact on pregnancy management and outcome. BMC Pregnancy Childbirth 2018;18:1-13.

27 Bebber FE, Rizzolli J, Casagrande DS, et al. Pregnancy after bariatric surgery: 39 pregnancies follow-up in a multidisciplinary team. Obes Surg 2011;21:1546-51.

28 Devlieger R, Guelinckx I, Jans G, et al. Micronutrient levels and supplement intake in pregnancy after bariatric surgery: a prospective cohort study. PLoS One 2014;9:e114192.

29 Kloss O, Eskin NAM, Suh M. Thiamin deficiency on fetal brain development with and without prenatal alcohol exposure. Biochem Cell Biol 2018;96:169-77.

30 Janbek J, Specht IO, Heitmann BL. Associations between vitamin D status in pregnancy and offspring neurodevelopment: a systematic literature review. Nutr Rev 2019;77:330-49.

31 Hague WM. Homocysteine and pregnancy. Best Pract Res Clin Obstet Gynaecol 2003;17:459-69.

32 Steegers-Theunissen RPM, Steegers EAP. Nutrient-gene interactions in early pregnancy: a vascular hypothesis. Elsevier, 2003.

33 Ebisch IMW, Thomas CMG, Peters WHM, et al. The importance of folate, zinc and antioxidants in the pathogenesis and prevention of subfertility. Hum Reprod Update 2007;13:163-74.

34 Picciano MF. Is homocysteine a biomarker for identifying women at risk of complications and adverse pregnancy outcomes? Oxford University Press, 2000.

35 Roth DE. Vitamin D supplementation during pregnancy: safety considerations in the design and interpretation of clinical trials. $J$ Perinatol 2011;31:449-59.

36 Hales CN, Barker DJ, Clark PM, et al. Fetal and infant growth and impaired glucose tolerance at age 64. BMJ 1991;303:1019-22.

37 Dhana K, Haines J, Liu G. Association between maternal adherence to healthy lifestyle practices and risk of obesity in offspring: results from two prospective cohort studies of mother-child pairs in the United States. BMJ 2018;362:k2486.

38 Dhana K, Zong G, Yuan C, et al. Lifestyle of women before pregnancy and the risk of offspring obesity during childhood through early adulthood. Int J Obes 2018;42:1275-84.

39 Barker DJ. The fetal and infant origins of adult disease. BMJ 1990;301:1111.

40 Ley RE, Bäckhed F, Turnbaugh $\mathrm{P}$, et al. Obesity alters gut microbial ecology. Proc Natl Acad Sci U S A 2005;102:11070-5.

41 Yadav H, Jain S, Nagpal R, et al. Increased fecal viral content associated with obesity in mice. World J Diabetes 2016;7:316.

42 Knight AK, Park HJ, Hausman DB, et al. Association between onecarbon metabolism indices and DNA methylation status in maternal and cord blood. Sci Rep 2018;8:1-9.

43 Ducker GS, Rabinowitz JD. One-carbon metabolism in health and disease. Cell Metab 2017;25:27-42.

44 Farin W, Oñate FP, Plassais J, et al. Impact of laparoscopic Rouxen-Y gastric bypass and sleeve gastrectomy on gut microbiota: a metagenomic comparative analysis. Surg Obes Relat Dis 2020;16:852-62.

45 Steegers-Theunissen RP, Verheijden-Paulissen JJ, van Uitert EM, et al. Cohort profile: the Rotterdam periconceptional cohort (predict study). Int J Epidemiol 2016;45:374-81.

46 Thompson FE, Byers T. Dietary assessment resource manual. J Nutr 1994; $124: 2245 s-317$.

47 Cade J, Thompson R, Burley V, et al. Development, validation and utilisation of food-frequency questionnaires - a review. Public Health Nutr 2002;5:567-87.

48 Steinemann N, Grize L, Ziesemer K, et al. Relative validation of a food frequency questionnaire to estimate food intake in an adult population. Food Nutr Res 2017;61:1305193.

49 Basbug A, Ellibeș Kaya A, Dogan S, et al. Does pregnancy interval after laparoscopic sleeve gastrectomy affect materna and perinatal outcomes? J Matern Fetal Neonatal Med 2019;32:3764-70.

50 Costa MM, Belo S, Souteiro P, et al. Pregnancy after bariatric surgery: maternal and fetal outcomes of 39 pregnancies and a literature review. J Obstet Gynaecol Res 2018;44:681-90.

51 Coupaye M, Legardeur H, Sami O, et al. Impact of Roux-en-Y gastric bypass and sleeve gastrectomy on fetal growth and relationship with maternal nutritional status. Surg Obes Relat Dis 2018;14:1488-94. 
52 De Carolis S, Botta A, Del Sordo G, et al. Influence of biliopancreatic diversion on pregnancy outcomes in comparison to other bariatric surgery procedures. Obes Surg 2018;28:3284-92.

53 Hazart J, Le Guennec D, Accoceberry M, et al. Maternal nutritional deficiencies and small-for-gestational-age neonates at birth of women who have undergone bariatric surgery. $J$ Pregnancy 2017;2017:1-11.

54 Nilsson-Condori E, Hedenbro JL, Thurin-Kjellberg A, et al. Impact of diet and bariatric surgery on anti-Müllerian hormone levels. Hum Reprod 2018;33:690-3.

55 Parent B, Martopullo I, Weiss NS, et al. Bariatric surgery in women of childbearing age, timing between an operation and birth, and associated perinatal complications. JAMA Surg 2017;152:128-35.

56 Rasteiro C, Araújo C, Cunha S, et al. Influence of time interval from bariatric surgery to conception on pregnancy and perinatal outcomes. Obes Surg 2018;28:3559-66.
57 Kabbi MSA, Al-Taee HA, Kareem Al Hussaini S. Impact of bariatric surgery on antimularian hormone in reproductive age women. Middle East Fertil Soc J 2018

58 Vincentelli C, Maraninchi M, Valéro R, et al. One-year impact of bariatric surgery on serum anti-Mullerian-hormone levels in severely obese women. J Assist Reprod Genet 2018;35:1317-24.

59 Yau PO, Parikh M, Saunders JK, et al. Pregnancy after bariatric surgery: the effect of time-to-conception on pregnancy outcomes. Surg Obes Relat Dis 2017;13:1899-905.

60 Van Dijk MR, Borggreven NV, Willemsen SP, et al. Maternal lifestyle impairs embryonic growth: the Rotterdam Periconception cohort. Reprod Sci 2018;25:916-22.

61 Nybo Andersen AM, Wohlfahrt J, Christens P, et al. Maternal age and fetal loss: population based register linkage study. BMJ 2000;320:1708-12. 